\title{
MRI-guided stereotactic laser corpus callosotomy for epilepsy: distinct methods and outcomes
}

\author{
Christopher W. Rich, MD, ${ }^{1}$ Rebecca E. Fasano, MD, ${ }^{2}$ Faical Isbaine, PhD, ${ }^{3}$ Amit M. Saindane, MD, ${ }^{4}$ \\ Deqiang Qiu, PhD, ${ }^{4}$ Daniel J. Curry, MD, ${ }^{5}$ Robert E. Gross, MD, PhD, ${ }^{3}$ and Jon T. Willie, MD, $\mathrm{PhD}{ }^{3,6}$
}

${ }^{1}$ Emory University School of Medicine; Departments of ${ }^{2}$ Neurology, ${ }^{3}$ Neurosurgery, and ${ }^{4}$ Radiology, Emory University School of Medicine, Atlanta, Georgia; ${ }^{5}$ Department of Neurosurgery, Texas Children's Hospital, Houston, Texas; and ${ }^{6}$ Department of Neurological Surgery, Washington University School of Medicine, St. Louis, Missouri

\begin{abstract}
OBJECTIVE Several small series have described stereotactic MRI-guided laser interstitial thermal therapy for partial callosotomy of astatic and generalized tonic-clonic (GTC) seizures, especially in association with Lennox-Gastaut syndrome. Larger case series and comparison of distinct stereotactic methods for stereotactic laser corpus callosotomy (SLCC), however, are currently lacking. The objective of this study was to report seizure outcomes in a series of adult patients with epilepsy following anterior, posterior, and complete SLCC procedures and to compare the results achieved with a frameless stereotactic surgical robot versus direct MRI guidance frames.
\end{abstract}

METHODS The authors retrospectively reviewed sequential adult epilepsy surgery patients who underwent SLCC procedures at a single institution. They describe workflows, stereotactic errors, percentage disconnection, hospitalization durations, adverse events, and seizure outcomes after performing anterior, posterior, and complete SLCC procedures using a frameless stereotactic surgical robot versus direct MRI guidance platforms.

RESULTS Thirteen patients underwent 15 SLCC procedures. The median age at surgery was 29 years (range 20-49 years), the median duration of epilepsy was 21 years (range 9-48 years), and median postablation follow-up was 20 months (range 4-44 months). Ten patients underwent anterior SLCC with a median $73 \%$ (range 33\%-80\%) midsagittal length of callosum acutely ablated. Following anterior SLCC, 6 of 10 patients achieved meaningful (> 50\%) reduction of target seizures. Four patients underwent posterior (completion) SLCC following prior anterior callosotomy, and 1 patient underwent complete SLCC as a single procedure; 3 of these 5 patients experienced meaningful reduction of target seizures. Overall, 8 of 10 patients in whom astatic seizures were targeted and treated by anterior and/or posterior SLCC experienced meaningful improvement. SLCC procedures with direct MRI guidance $(n=7)$ versus a frameless surgical robot $(n=8)$ yielded median radial accuracies of $1.1 \mathrm{~mm}$ (range $0.2-2.0 \mathrm{~mm}$ ) versus $2.4 \mathrm{~mm}$ (range $0.6-6.1 \mathrm{~mm} ; p=$ 0.0011). The most serious adverse event was a clinically significant intraparenchymal hemorrhage in a patient who underwent the robotic technique.

CONCLUSIONS This is the largest reported series of SLCC for epilepsy to date. SLCC provides seizure outcomes comparable to open surgery outcomes reported in the literature. Direct MRI guidance is more accurate, which has the potential to reduce the risks of SLCC. Methodological advancements and larger studies are needed.

https://thejns.org/doi/abs/10.3171/2020.7.JNS20498

KEYWORDS stereotactic laser ablation; laser interstitial thermal therapy; MRI; surgical robotics; corpus callosotomy; Lennox-Gastaut syndrome; generalized epilepsy; astatic seizures; atonic seizures; drop attacks

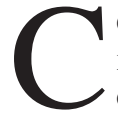
ORPUS callosotomy, which inhibits interhemispheric spread of seizures, has been practiced in selected patients with debilitating motor seizures since the 1940s. ${ }^{1,2}$ Anterior callosotomy including the isthmus ${ }^{3}$ is widely considered the first stage of callosotomy, with subsequent complete division (including the splenium) re- served for patients with continuation of the targeted seizures. ${ }^{4}$ Callosotomy is an effective treatment of astatic (including tonic, atonic, and otherwise unspecified "drop attack") seizures, which meta-analyses suggest to be superior to vagus nerve stimulation (VNS). ${ }^{5}$ Open anterior two-thirds callosotomy and complete callosotomy are ex-

ABBREVIATIONS DBS = deep brain stimulation; GTC = generalized tonic-clonic; LGS = Lennox-Gastaut syndrome; LOS = length of stay; MRg-LITT = MRI-guided laser interstitial thermal therapy; ROSA = Robotic Stereotactic Assistant; SGE = symptomatic generalized epilepsy; SLCC = stereotactic laser corpus callosotomy; T2IR = T2 inversion recovery; VNS = vagus nerve stimulation; $V P=$ ventriculoperitoneal.

SUBMITTED February 18, 2020. ACCEPTED July 15, 2020.

INCLUDE WHEN CITING Published online January 22, 2021; DOI: 10.3171/2020.7.JNS20498. 
pected to provide greater than $50 \%$ reduction in drop attacks in $45 \%$ and $90 \%$ of patients, respectively, based on systematic review of outcomes in pediatric patients. ${ }^{6-10}$

Open corpus callosotomy is usually performed via frontal craniotomy and microscopic interhemispheric dissection. ${ }^{11}$ Reported surgical complications include intracranial hemorrhage, retraction injury, venous infarcts, bone flap osteomyelitis, subgaleal fluid collection, hydrocephalus, and sagittal sinus tearing, ${ }^{6,12-14}$ and hospitalizations and recovery periods can be lengthy. Open posterior completion callosotomy can yield additional seizure benefit but carries added risks of vascular injuries and neurological deficits. In addition, persistent symptomatic disconnection syndrome is more likely in patients following total corpus callosotomy (15.4\%) compared with anterior two-thirds corpus callosotomy (6.9\%); ${ }^{6}$ thus, less invasive approaches are desirable.

Stereotactic MRI-guided laser interstitial thermal therapy (MRg-LITT) is an emerging minimally invasive alternative to open epilepsy surgery, especially for focal and deep lesions. ${ }^{15-20}$ Stereotactic laser corpus callosotomy (SLCC) based on MRg-LITT was successfully performed first by Curry (personal communication, November 2013) and further described in a handful of subsequent case reports and small series. ${ }^{21-28}$ MRg-LITT procedures can be performed by various stereotactic techniques, but direct comparison of approaches is lacking.

In this paper, we report on 13 adult patients who underwent 15 SLCC procedures, the largest sequential series at a single surgical epilepsy center. These procedures included anterior ("two-thirds") SLCC, posterior completion SLCC after prior open or laser anterior callosotomy, and a complete SLCC performed as a single procedure. In addition, we directly compare the stereotactic accuracies of two approaches: direct MRI guidance (ClearPoint Neuro, Inc.) versus stereotactic bolts placed by stereotactic surgical robot (Robotic Stereotactic Assistant [ROSA], Zimmer Biomet, Inc.).

\section{Methods \\ Study Population}

This retrospective study was approved by the Emory University IRB. A search of a spreadsheet database of all adult epilepsy surgery patients treated by two surgeons (J.T.W. and R.E.G.) was used to identify those who had undergone SLCC by either surgeon. Medical records were reviewed for age, sex, epilepsy duration, typical seizure types and frequencies, EEG results, treatment history, MRI brain results, seizure types targeted for therapy (e.g., astatic vs generalized tonic-clonic [GTC] seizures), durations of surgery and hospitalization, adverse events, and seizure outcomes.

\section{Surgical Methods}

All MRg-LITT utilized the Visualase Thermal Therapy System (Medtronic, Inc.). ${ }^{18,29}$ The two distinct workflows utilized for stereotactic techniques in this study are illustrated in the Supplemental Figure. The earliest cases were performed by direct MRI-guided technique (ClearPoint Neuro, Inc.). When we subsequently obtained a stereotactic surgical robot (ROSA), we began using it for cases with multiple trajectories (anterior callosotomies), expecting to possibly reduce the procedure time. Choice of approach was determined by this consideration rather than patient selection. In contrast, we performed all posterior completion callosotomies using the direct MRIguided technique, as there was no anticipated benefit of the robotic technique for single-trajectory cases. ROSA procedures involve imaging coregistration by methods including either scalp surface reflectance or bone fiducials. In other ROSA procedures (stereoelectroencephalography), we found that bone fiducials did not benefit accuracy, so all our ROSA SLCC procedures utilized scalp surface coregistration.

\section{MRg-LITT by Direct MRI-Guided Technique}

Patients underwent endotracheal general anesthesia in an interventional MRI suite (1.5-T Espree, Siemens, Inc.) and were positioned supine with shoulder bump and lateral head turn in pins and flanked by flexible radiofrequency receive-only head coils (Fig. 1). After sterile scalp preparation and draping, adhesive fiducial grids were placed over the approximate entry sites at nondominant parasagittal frontal and parietal locations. Volumetric imaging, consisting of T2 inversion recovery (T2IR)-weighted and gadolinium-enhanced (postcontrast) 3D T1-weighted MRI, was obtained and used to plan trajectories with a stereotactic workstation (ClearPoint Neuro, Inc.).

For anterior callosotomies, we planned 3-4 trajectories targeting the genu, anterior body, and posterior body with isthmus (Fig. 1). Entries were $2-3 \mathrm{~cm}$ off the midline, passed through cingulate structures, pierced the callosum within $1 \mathrm{~cm}$ from midline, and terminated at or just beyond the midline callosum. We planned a margin of 4-6 $\mathrm{mm}$ in diameter to avoid visible blood vessels. For posterior completion callosotomies, only the remaining splenium was targeted, avoiding the fornices and deep cerebral veins (Fig. 2). Based on an assumed ablation diameter estimate of approximately $18 \mathrm{~mm}$, trajectories were planned to confluently ablate the anteroposterior parasagittal extent of the targeted portion of the corpus callosum.

Fiducial grids were used to mark final entry sites on the scalp, and overlying ClearPoint "Scalpmount" base frames were affixed percutaneously to the skull with lag screws and stabilizing offset pins. Subsequent imaging was iteratively processed by the ClearPoint workstation to provide prescriptions to align each platform to the desired trajectory using $\mathrm{x}, \mathrm{y}$, pitch, and roll adjustments. For each trajectory, we performed a stereotactic 3.2-mm twist-hole craniostomy with a hand drill via a stab incision, realigned the frame to the desired trajectory as needed, and punctured the dura. A ceramic stylet was placed to target depth and its location was verified with imaging. The ceramic stylet was replaced with the stiffened Visualase cannula, and then the optical fiber with a 10 -mm diffusing tip was placed within the cannula and irrigating tubing was attached. MR-based thermal imaging was obtained in two planes along the length of the cannula. Using real-time thermal imaging and the Visualase workstation, a series of confluent ablations $1 \mathrm{~cm}$ in length were created through the corpus callosum along each trajectory. We strove to 
Rich et al.

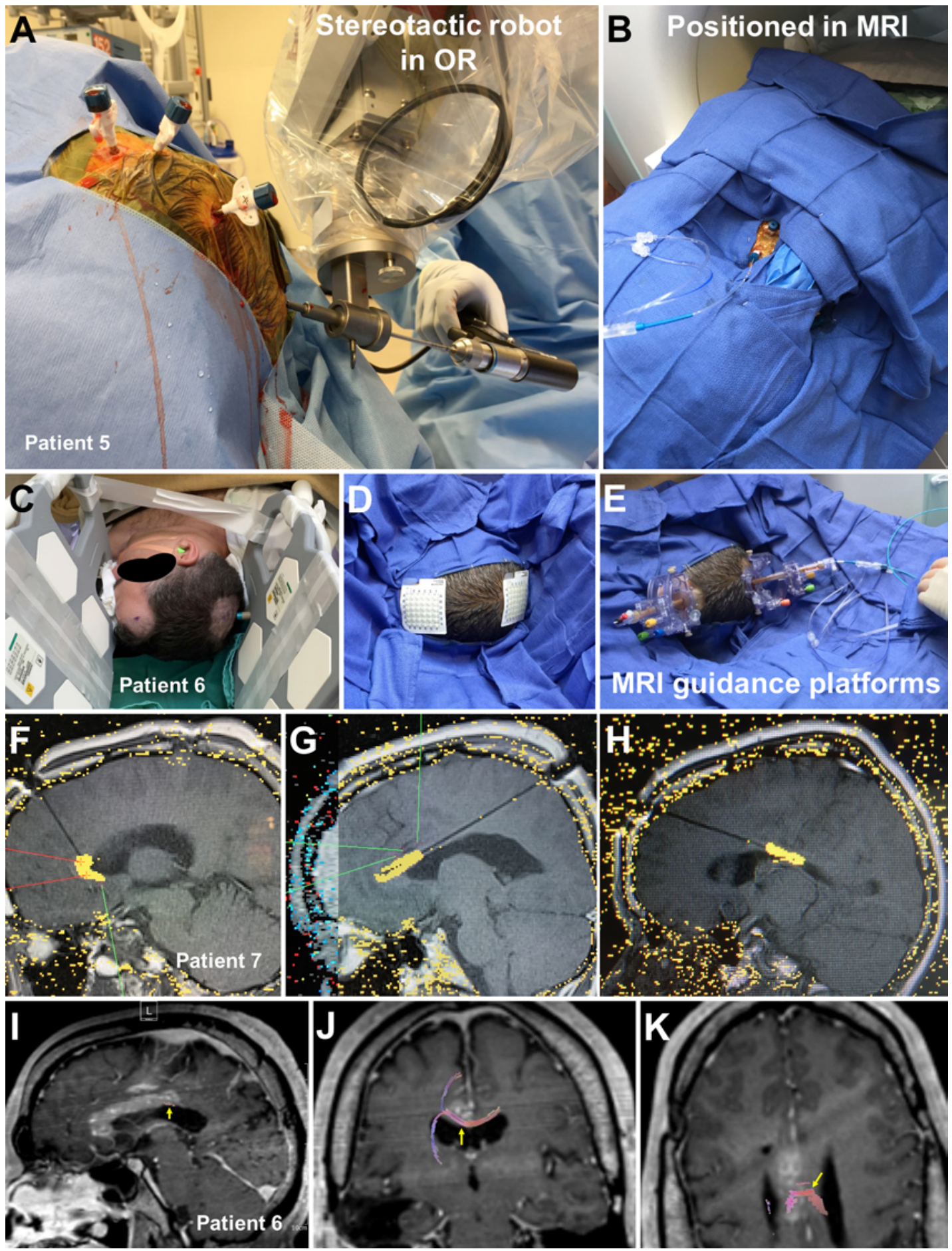

FIG. 1. Alternative surgical approaches for anterior two-thirds SLCC. A: Intraoperative photograph of patient 5 undergoing drilling and placement of bolts with a stereotactic surgical robot (ROSA). B: Patient 5 positioned and draped in a 1.5-T interventional MRI machine with a stereotactic bolt holding the Visualase laser interstitial thermal therapy system. C: Patient 6 positioned supine with head turned laterally and pinned to expose nondominant hemisphere for direct MRI guidance. The head is flanked by flexible MRI head coils. D: Patient's head prepped and draped with MRI-compatible fiducial grids over intended entry sites. E: Disposable MRI guidance platforms are mounted overlying desired entry sites with percutaneous screws. A frame is aligned with imaging, a percutaneous twist-drill craniotomy is performed, and the Visualase laser cannula is placed stereotactically to the target. F-H: Visualase workstation screenshots of parasagittal T1 anatomical views merged with real-time thermal damage maps (orange pixels) along the genu $(\mathrm{F})$, body $(\mathrm{G})$, and isthmus $(\mathrm{H})$ of the corpus callosum in patient 7. I-K: Sagittal $(\mathrm{I})$, coronal $(\mathrm{J})$, and axial $(\mathrm{K})$ T1-weighted contrast-enhanced MR images of patient 6 showing the extent of ablation relative to coregistered diffusion imaging models of fiber pathways (colored fibers emphasized with yellow arrows) seeded from the region of the central sulcus (primary motor pathways) 

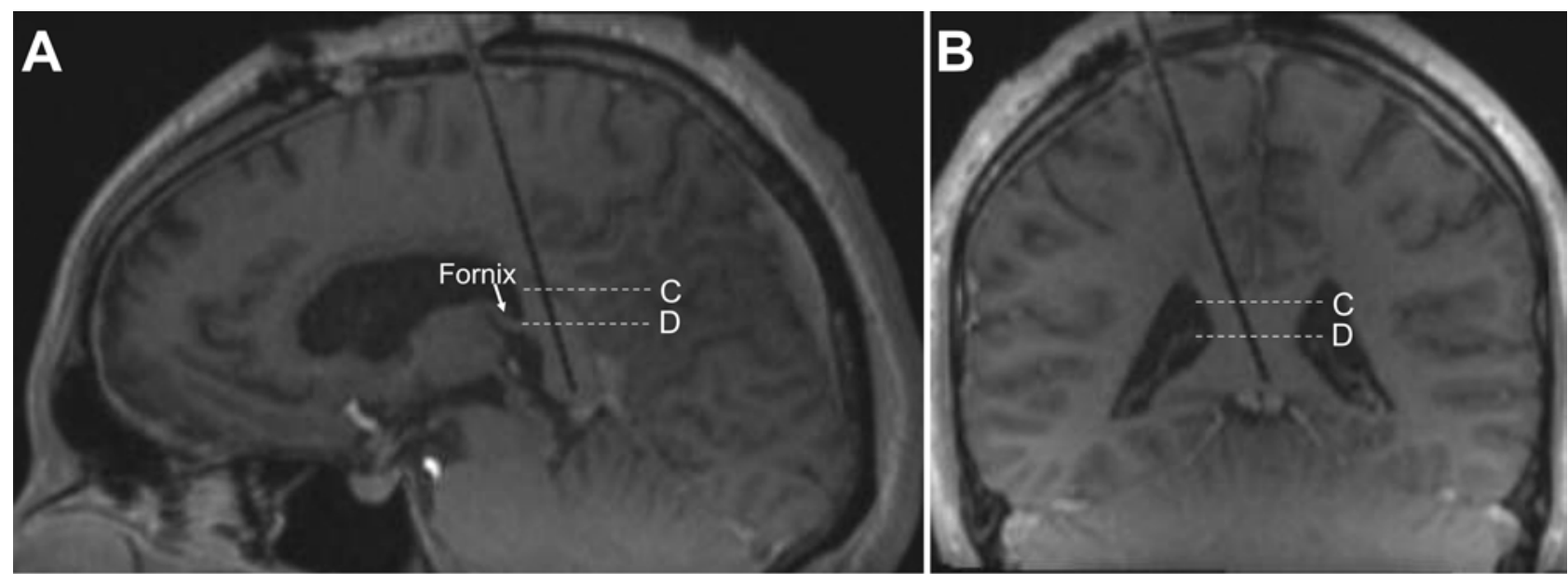

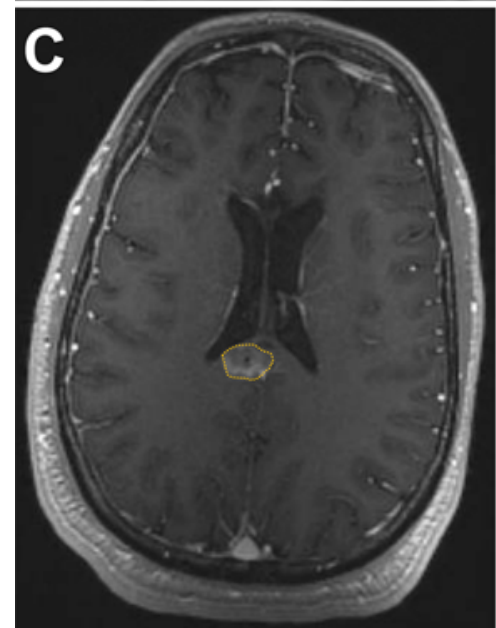

$\mathbf{F}$
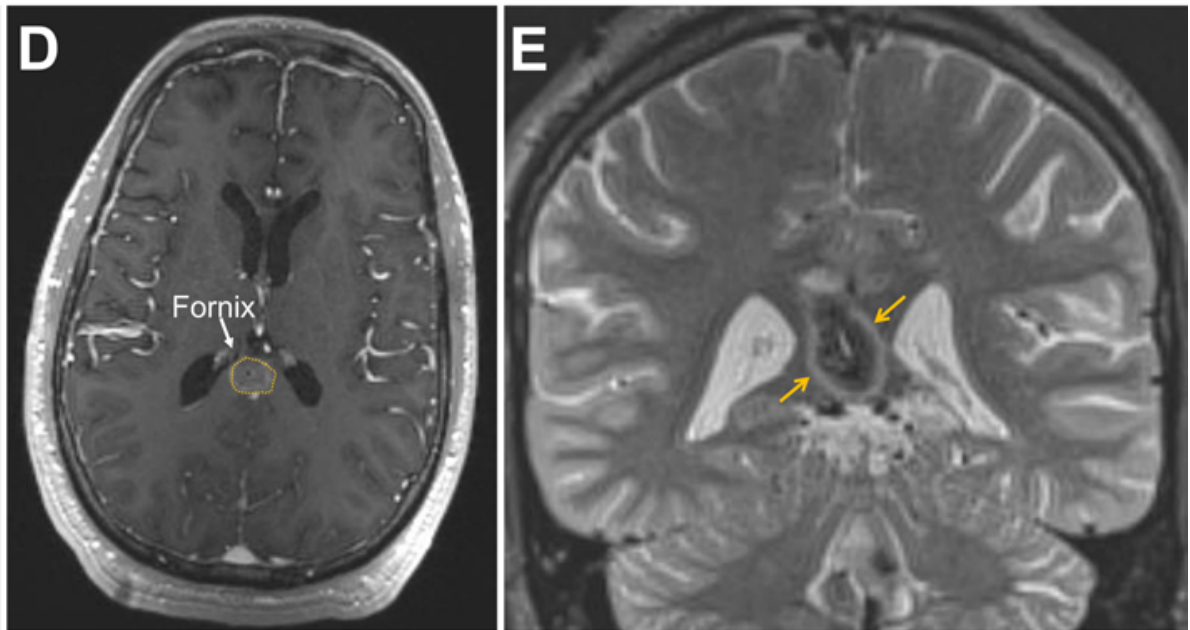

\section{Post-ablation}

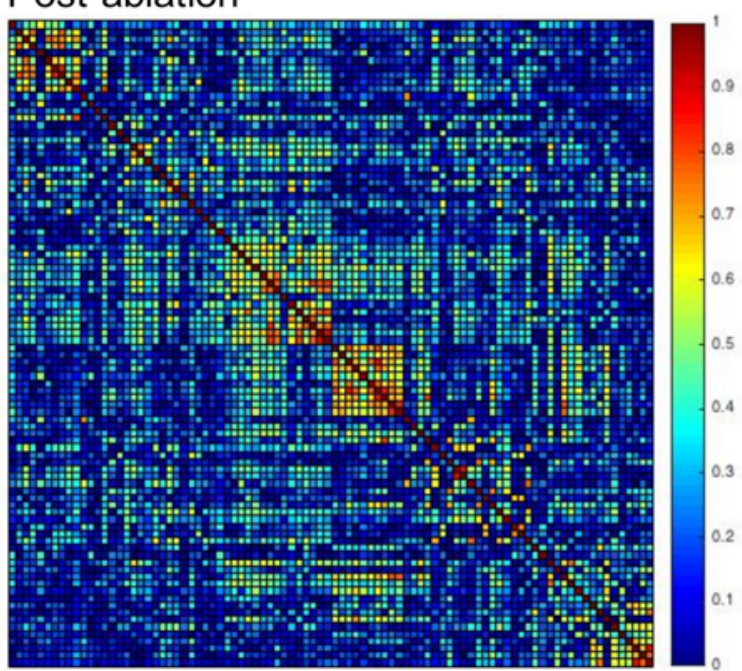

FIG. 2. Posterior completion SLCC of the splenium. An MRI guidance platform (ClearPoint Neuro, Inc.) was used to ablate the remaining splenium to complete the callosotomy in a patient with recurrent atonic seizures after an initially successful open anterior corpus callosotomy. A and B: Parasagittal (A) and paracoronal (B) T1-weighted MR images of laser device placement in the splenium. Note the proximity of the fornix (labeled), the ablation of which should be avoided. Dashed white lines indicate the locations of the subsequent axial images. C and D: Axial T1-weighted MR images that demonstrate full anterior-posterior ablation (dotted orange outlines) of the splenium and proximity to the fornix. E: Coronal inversion recovery MR image demonstrates ablation disconnecting the full superior-inferior extent of the splenium (arrows). F: Pre- and postablation resting-state functional connectivity MRI results under similar levels of inhaled anesthetic show reduced hemispheric connectivity immediately following posterior completion callosotomy. The heat map uses the color red to represent the greatest level of connectivity between two locations. 
avoid thermal injury of cortices outside the callosum (i.e., cingulum) using low-temperature thermal limits. More than one trajectory could typically be performed by simply realigning the frame to a new trajectory and drilling a new craniostomy. Because a sterile field was maintained, a single laser apparatus was typically reused for multiple trajectories. FLAIR, diffusion, T2IR, and postcontrast T1weighted MRI were used to verify confluent ablations.

\section{MRg-LITT by Frameless Stereotactic Robot-Assisted Technique}

Under endotracheal general anesthesia in the operating room, patients were positioned supine with the head in a Radionics CRW (Integra, Inc.) universal head holder frame base, which was then attached using a Mayfield adapter (Integra, Inc.) to the stereotactic surgical robot (ROSA; Fig. 1). Using prior volumetric CT and gadolinium-enhanced MRI, trajectories were planned as described in the previous section, but with ROSA planning software. After reflectance-based coregistration of the skin surface to historical CT and MR imaging, and sterile preparation and draping, a 3.2-mm twist-hole craniostomy was performed through a stab incision for each trajectory under stereotactic control of the robotic arm. Durotomies were completed with a stylet, and titanium Visualase bolts were screwed into each craniostomy over an aligning rod using robotic stereotactic control. The bolts and surrounding sterile field were sealed under nonpermeable adhesive drapes (Tegaderm and Ioban), and the patient was transported to the interventional MRI suite where a sterile field was recovered by removing the drape to expose a bolt that was resterilized with betadine. The stiffened Visualase cannula was inserted to depth through a bolt, the laser fiber and coolant cannula were assembled, and MRI was performed to verify placement. After each ablation trajectory, with the sterile field maintained, the apparatus was withdrawn, inspected, and then used through the next bolt. Thus, for most cases, a single laser apparatus was used to complete all ablation trajectories.

\section{Data Analysis and Literature Review}

Radial error, a standard measure of stereotactic accuracy, is the difference (perpendicular distance) between the center of the intended trajectory at the target to the center of the actual device placement on subsequent imaging. ${ }^{30}$ We retrospectively measured radial errors at the target using T1-weighted MRI from 17 of 25 recoverable ROSA trajectories and 14 of 15 recoverable ClearPoint trajectories that had remained saved in the respective workstations. To assess the extent of callosum ablated in each procedure, we measured the internal perimeter length of the callosum from the inferior aspect of the genu to the inferior aspect of the splenium, and calculated the analogous length of ablation as a percentage of the total.

Although callosotomy may benefit multiple seizure types, ${ }^{5,27,31}$ we report outcomes with respect to the targeted astatic and GTC seizures as resolved (no further documented seizures of the targeted subtype), meaningfully improved ( $>50 \%$ reduction but not completely resolved), or not meaningfully improved $(<50 \%$ reduction).
We separately report these results for 1) anterior SLCC, 2) posterior SLCC (completion following prior anterior callosotomy by either open or laser approach), and 3) complete SLCC.

For statistical analysis, two nonparametric tests were used for assessment of significance. Comparison of all continuous data was completed using the Mann-Whitney U-test, where sample sizes were more than 5 and $p$ values were generated following conversion to a z-score. This included comparison of ClearPoint and ROSA methods with respect to trajectories required, percentage of length of callosum ablated, and lengths of stay (LOSs). The Fisher exact test was used for categorical variables (seizure outcomes, adverse events, and persistent sequelae) when sample sizes were larger than 2 .

A literature review of MRg-LITT of the corpus callosum for epilepsy was performed by a PubMed search combining the search terms "corpus callosotomy" and "laser interstitial thermal therapy." Seven identified studies were reviewed for preoperative diagnosis, epilepsy duration, prior VNS therapy, duration of hospitalization, complications, and outcomes as defined by the authors of each individual study.

\section{Results}

\section{Patient Characteristics}

From 2014 to 2018, 13 adult patients underwent 15 SLCC procedures. Specifically, 10 patients underwent anterior SLCC, 1 (case 8) underwent complete SLCC, 2 (cases 2 and 11) underwent posterior SLCC following prior anterior SLCC, and 2 (cases 1 and 4) underwent posterior SLCC following prior remote open anterior corpus callosotomy. Nine patients carried a diagnosis of symptomatic generalized epilepsy (SGE; including Lennox-Gastaut syndrome [LGS]), and 4 patients were diagnosed as having poorly localized focal epilepsy with rapidly generalizing features (Table 1). Astatic seizures were the most common indication, although 3 patients (cases 2, 5, and 11) underwent SLCC to palliate GTC seizures. The median age at the time of surgery was 29 years (range $20-49$ years). The median duration of epilepsy at time of the procedure was 21 years (range $9-48$ years).

Many patients underwent multiple surgical procedures (Tables 1 and 2). The majority (all but cases 5, 7, 8, and 10) underwent prior VNS. Four patients underwent posterior SLCC after a prior anterior callosotomy. One such patient with LGS (case 1) who had undergone remote open anterior callosotomy also underwent left stereotactic laser amygdalohippocampotomy (MRg-LITT) for mesial temporal sclerosis, and then 1 year later underwent posterior (completion) SLCC for recurrent astatic seizures. One patient (case 5) underwent prior open anterior temporal lobectomy before undergoing posterior SLCC to palliate persistent secondary GTC seizures. After separate anterior and posterior SLCC procedures, case 11 subsequently underwent bilateral centromedian thalamic nucleus deep brain stimulation (DBS). Case 13 underwent craniotomy, cranioplasty, and ventriculoperitoneal (VP) shunt placement to manage a hemorrhagic complication of SLCC (described below). 


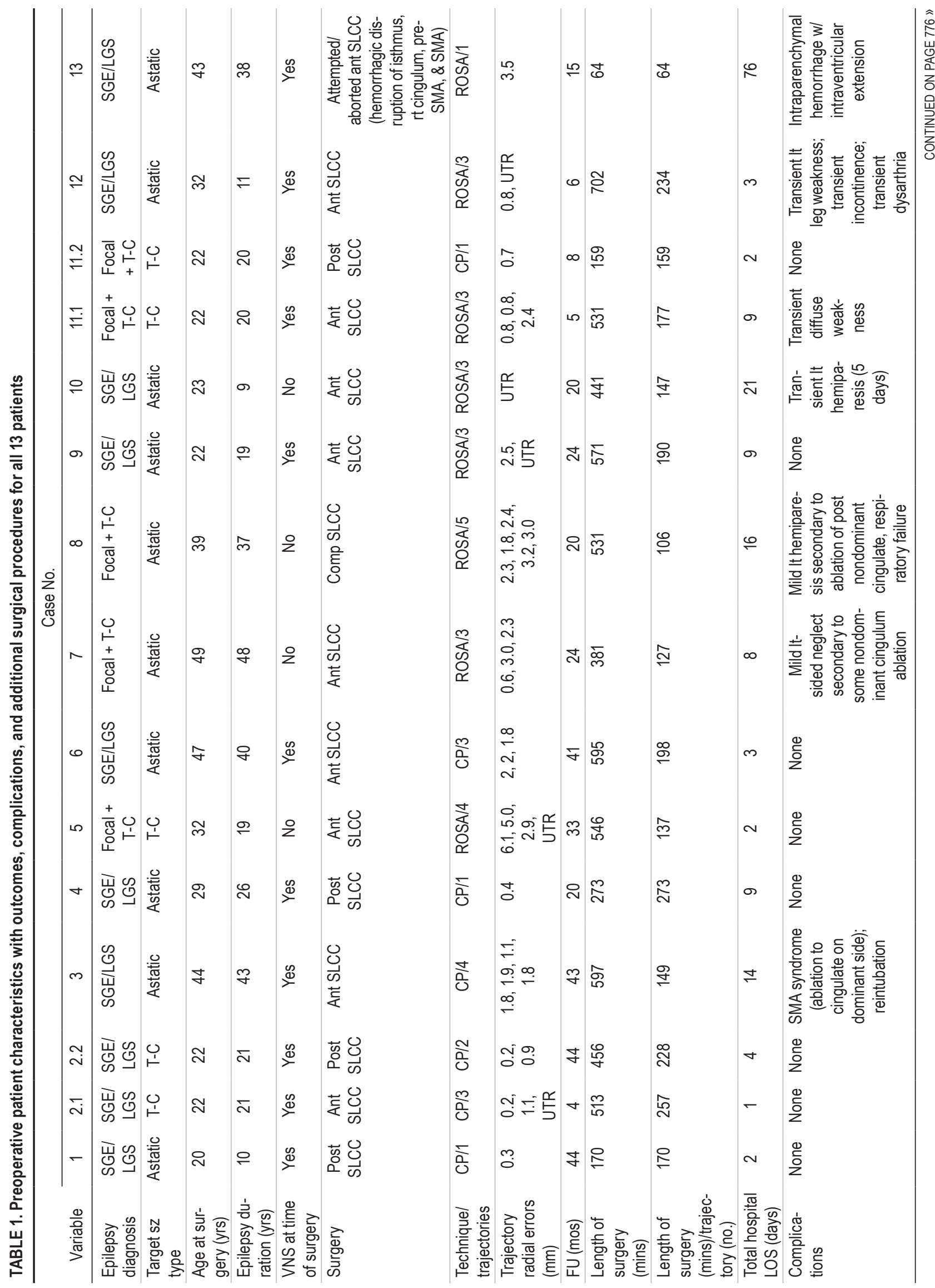




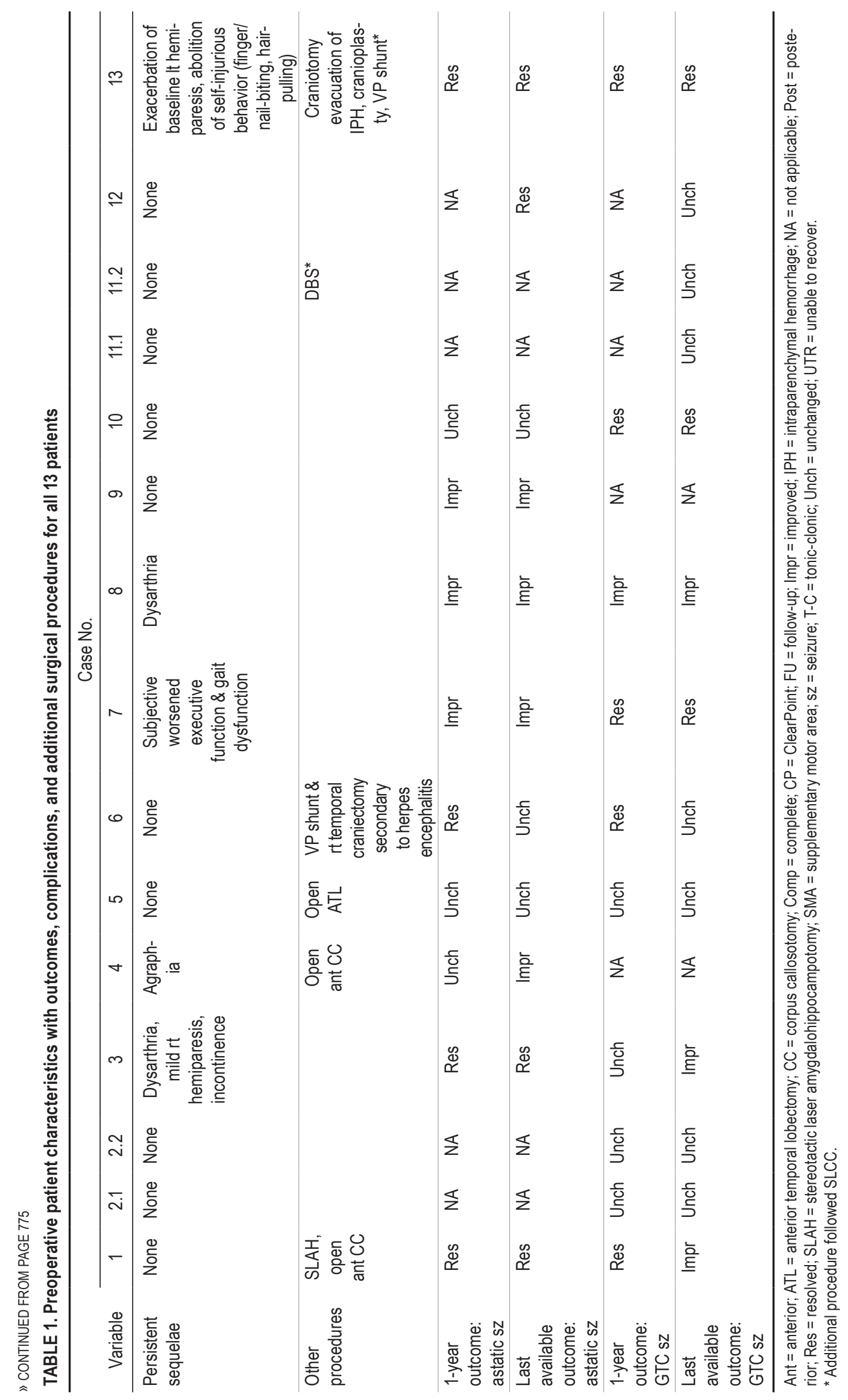


TABLE 2. Cases grouped by procedure type

\begin{tabular}{|c|c|c|c|c|}
\hline \multirow[b]{2}{*}{ Variable } & \multicolumn{4}{|c|}{ Procedure Type } \\
\hline & All SLCC & Anterior SLCC* & Posterior SLCC (completion) & Complete SLCC $3 / 3$ \\
\hline No. of cases & 15 & 10 & 4 & 1 \\
\hline Median length of procedure (range), mins & $513(64-702)$ & $539(64-702)$ & $222(159-456)$ & 531 \\
\hline No. of median trajectories (range) & $3(1-5)$ & $3(1-4)$ & $1(1-2)$ & 5 \\
\hline Median follow-up (range), mos & $20(4-44)$ & $22(4-43)$ & $32(8-44)$ & 20 \\
\hline Median total hospital LOS (range), days & $8(1-76)$ & $9(1-76)$ & $3(3-9)$ & 16 \\
\hline Cases w/ adverse events & 7 & 6 & 0 & 1 \\
\hline Cases w/ persistent sequelae & 5 & 3 & 1 & 1 \\
\hline Estimated collosum length ablated & $78 \%(33-100 \%)$ & $73 \%(33-80 \%)$ & $100 \%(98-100 \%)$ & $100 \%$ \\
\hline \multicolumn{5}{|l|}{ Outcome: targeted sz (no.) $\dagger$} \\
\hline Resolved & 3 & 3 & 1 & 0 \\
\hline Improved & 5 & 3 & 1 & 1 \\
\hline \multicolumn{5}{|l|}{ Outcome: all szs (no.) } \\
\hline Resolved & 3 & 3 & 0 & 0 \\
\hline Improved & 2 & 1 & 1 & 1 \\
\hline
\end{tabular}

* Intention to treat.

† Targeted seizure type was either astatic or GTC (detailed in Table 1).

\section{SLCC Procedure Characteristics}

By intention-to-treat analysis, 10 patients underwent anterior SLCC procedures with a median of 3 trajectories (range 1-4 trajectories) completed (Table 1) and a median estimated $73 \%$ (range 33\%-80\%) of the callosal length ablated. One of these cases, an outlier (case 13), had only one trajectory, and the procedure was aborted due to hemorrhage (detailed below), achieving only an estimated $33 \%$ of the callosum disconnected (Table 2). The overall median duration of completed anterior SLCC procedures was 546 minutes (range 381-702 minutes), excluding the aborted case (case 13; Table 2). Anterior SLCC procedures were associated with a median acute hospital LOS of 9 days (range 1-76 days).

When three complete callosotomies were performed in either one or two stages, a total of 4-5 trajectories were used. For posterior SLCC procedures, a median of 1 trajectory (range 1-2 trajectories) was used to complete the callosotomy in all cases. The median duration of posterior completion SLCC procedures was 222 minutes (range 159-456 minutes). Posterior completion SLCC after a prior anterior procedure was associated with a median LOS of 3 days (range 3-9 days). The only complete SLCC performed in a single surgical session (case 8) had a duration of 531 minutes, and this procedure was associated with a 16-day LOS.

\section{Adverse Events}

Adverse events were found to be neurological or cardiorespiratory in nature and were categorized as transient or persistent. We observed no cranial infections or wound complications. Neurological symptoms such as weakness, neglect, abulia, or dysarthria were common but typically transient (Table 1) and may be attributed to acute disconnection. ${ }^{23,32}$ Seven of 15 SLCC procedures were associated with adverse events. Five patients exhibited persistent neu- rological complaints at last follow-up. Specifically, patient 3 was transiently reintubated for respiratory failure but had persistent mild right hemiparesis, dysarthria, and incontinence. Patient 4 reported persistent agraphia following posterior completion SLCC. Patient 7 reported worsening executive function and gait ataxia. Patient 8 , who underwent complete SLCC in a single surgical session, was transiently reintubated for respiratory failure and experienced transient hemiparesis followed by persistent dysarthria.

Patient 13 experienced a series of complications as a result of attempted anterior SLCC. Following placement of a laser device using ROSA, MRI revealed a $3.5-\mathrm{mm}$ device deviation from the intended trajectory and a right frontal hemorrhage in the dorsal cingulate region with intraventricular extension (Fig. 3). The procedure was aborted without any ablation, and the patient underwent
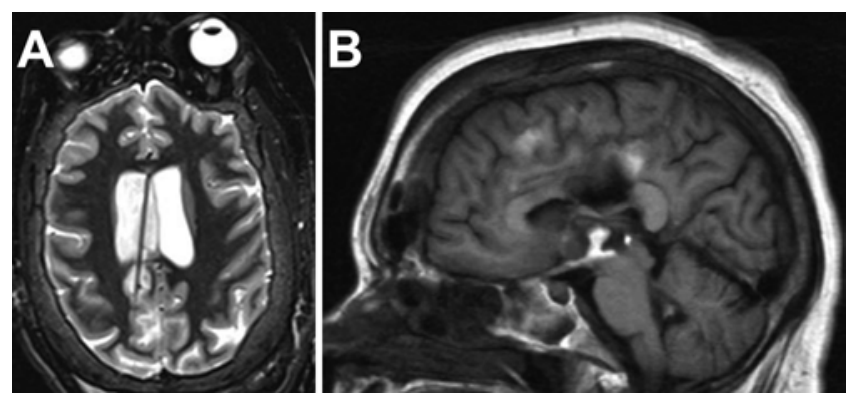

FIG. 3. Hemorrhagic complication of attempted SLCC. A and B: Axial (A) and sagittal (B) MR images from patient 13 in which initial placement of the laser cannula via a stereotactic robotic approach was associated with deflection and intraparenchymal hemorrhage with intraventricular extension. Following recovery, imaging revealed injury of the isthmus of the corpus callosum (B) as well as dorsal cingulum and medial frontal lobe white matter. The patient experienced exacerbation of baseline left hemiparesis in addition to complete seizure control. 
Rich et al.

TABLE 3. Comparison of SLCC techniques

\begin{tabular}{|c|c|c|c|c|c|c|c|c|}
\hline $\begin{array}{l}\text { SLCC } \\
\text { Method }\end{array}$ & $\begin{array}{c}\text { No. of } \\
\text { Procedures }\end{array}$ & $\begin{array}{l}\text { Length of } \\
\text { Procedure } \\
\text { (range), mins }\end{array}$ & $\begin{array}{l}\text { Length of Procedure/ } \\
\text { Trajectory (range), mins }\end{array}$ & $\begin{array}{c}\text { Total } \\
\text { Hospital LOS } \\
\text { (range), days }\end{array}$ & $\begin{array}{l}\text { No. of } \\
\text { Adverse } \\
\text { Events* }\end{array}$ & $\begin{array}{c}\text { No. of } \\
\text { Persistent } \\
\text { Sequelae }\end{array}$ & $\begin{array}{l}\text { No. of } \\
\text { Trajectories }\end{array}$ & $\begin{array}{c}\text { Median } \\
\text { Radial Error } \\
\text { (range), } \mathrm{mm}^{*}\end{array}$ \\
\hline \multicolumn{9}{|l|}{$\mathrm{CP}$} \\
\hline Ant & 3 & $595(513-597)$ & 198 (149-257) & $3(1-14)$ & 1 & 2 & 10 & $1.8(0.2-2)$ \\
\hline Post & 4 & $222(159-456)$ & $222(159-456)$ & $3(2-9)$ & 0 & 1 & 5 & $0.4(0.2-0.9)$ \\
\hline Total & 7 & $456(159-597)$ & 198 (149-273) & $2.5(1-14)$ & 1 & 3 & 15 (14 retrieved) & $1.1(0.2-2.0)$ \\
\hline \multicolumn{9}{|l|}{ ROSA } \\
\hline Ant & 6 & $539(381-702)$ & 162 (127-234) & $8.5(3-21)$ & 4 & 1 & 19 & $2.4(0.6-6.1)$ \\
\hline Comp & 1 & 531 & 106.2 & 16 & 1 & 1 & 5 & $2.4(1.8-3.2)$ \\
\hline Aborted & 1 & 64 & 64 & 76 & 1 & 1 & 1 & 3.5 \\
\hline Total & 8 & $531(381-702)$ & $147(106-234)$ & $9(2-76)$ & 6 & 3 & 25 (17 retrieved) & $2.4(0.6-6.1)$ \\
\hline
\end{tabular}

Statistical analysis was completed using two nonparametric tests for assessment of significance. All continuous data were analyzed using the Mann-Whitney $U$-test, where sample sizes were $>5$ and $p$ values were generated following conversion to a z-score. The Fisher exact test was used for categorical variables (seizure outcomes, adverse events, and persistent sequelae) where sample sizes were $>2$. No significance was found in variables outside of those annotated.

${ }^{*}$ Significant difference favoring ClearPoint found using $p<0.05$.

decompressive craniotomy to evacuate the hemorrhage. During the same prolonged hospital admission, she underwent tracheostomy, cranioplasty, and VP shunt placement. The patient suffered persistent exacerbation of baseline left hemiparesis, persistent injury of the right frontal lobe white matter, and division of the isthmus of the corpus callosum, which could explain subsequent resolution of clinical seizure activity.

Notably, despite the expectation that posterior callosotomy might increase the incidence of disconnection syndrome, only 1 of 4 posterior SLCC procedures (completion with ClearPoint) yielded a new neurological complaint.

\section{Seizure Outcomes}

Outcomes are categorized by targeted seizure type and procedure (Table 2). The median duration of followup was 20 months (range 4-44 months) for SLCC. Of 13 patients who underwent SLCC procedures, 10 suffered preoperatively from primarily astatic seizures, and all but 2 had GTCs as well. At the last available follow-up, 6 of 10 patients who underwent anterior SLCC experienced an improvement of more than $50 \%$ with respect to targeted seizures, and 4 of these patients exhibited complete resolution. Of the 7 patients with astatic seizures who underwent anterior SLCC, 5 of 7 were improved, with 3 of these experiencing complete seizure resolution. Notably, all 7 had improvement in at least one seizure type, with none showing exacerbation.

Of 5 patients who underwent posterior SLCC for persistent seizures or upfront complete SLCC, 3 experienced more than a 50\% meaningful benefit of targeted seizures, 1 of whom experienced resolution. All 3 patients with astatic seizures who underwent completion were improved, with 1 resolved. Improvement or resolution was achieved in 8 of 10 patients with primarily astatic seizures treated by anterior and/or posterior SLCC.

\section{ClearPoint Versus ROSA}

In general, the small numbers of cases performed by each stereotactic approach ( 7 by ClearPoint and 8 by ROSA) precluded meaningful statistical comparisons of procedure duration, hospital LOS, complications, and seizure outcomes (Table 3). However, the large number of trajectories performed in these cases provided the opportunity for meaningful comparison of stereotactic accuracy with each method. When compared on the basis of available trajectory data, 14 ClearPoint trajectories had a median radial error of $1.1 \mathrm{~mm}$ (range $0.2-1.9 \mathrm{~mm}$ ), while 17 ROSA trajectories had a larger median radial error of $2.4 \mathrm{~mm}$, with a considerably wider range of errors (range $0.7-6.1 \mathrm{~mm} ; \mathrm{p}=0.0011)$. Greater errors with ROSA suggest that it is a less accurate approach for performing callosotomy compared with ClearPoint (Table 3, Fig. 4). Notably, the most severe complication, an intraparenchymal hemorrhage in case 13, was associated with an inaccurate ROSA trajectory.

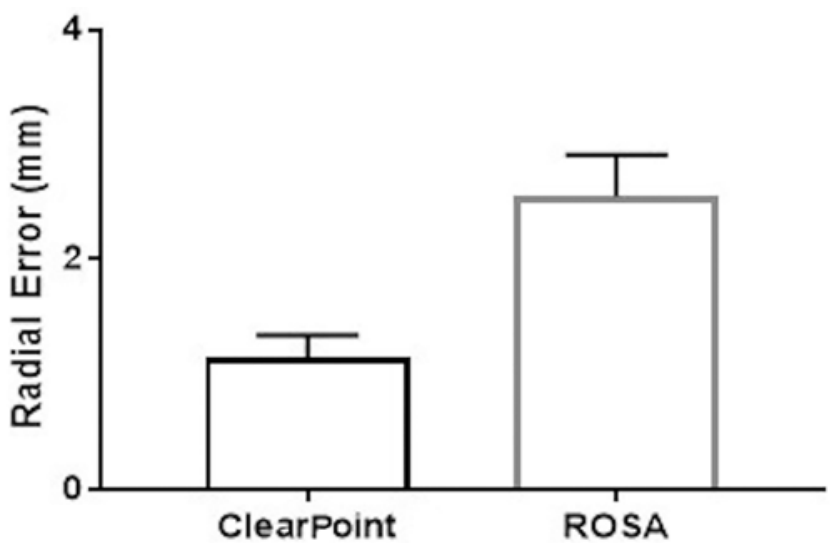

FIG. 4. Comparison analysis of trajectory radial errors obtained using direct MRI guidance platforms (ClearPoint) versus a stereotactic surgical robot (ROSA) for SLCC. The Mann-Whitney U-test was performed and found a significant reduction in radial error when using ClearPoint compared with ROSA ( $p=0.0011)$. 


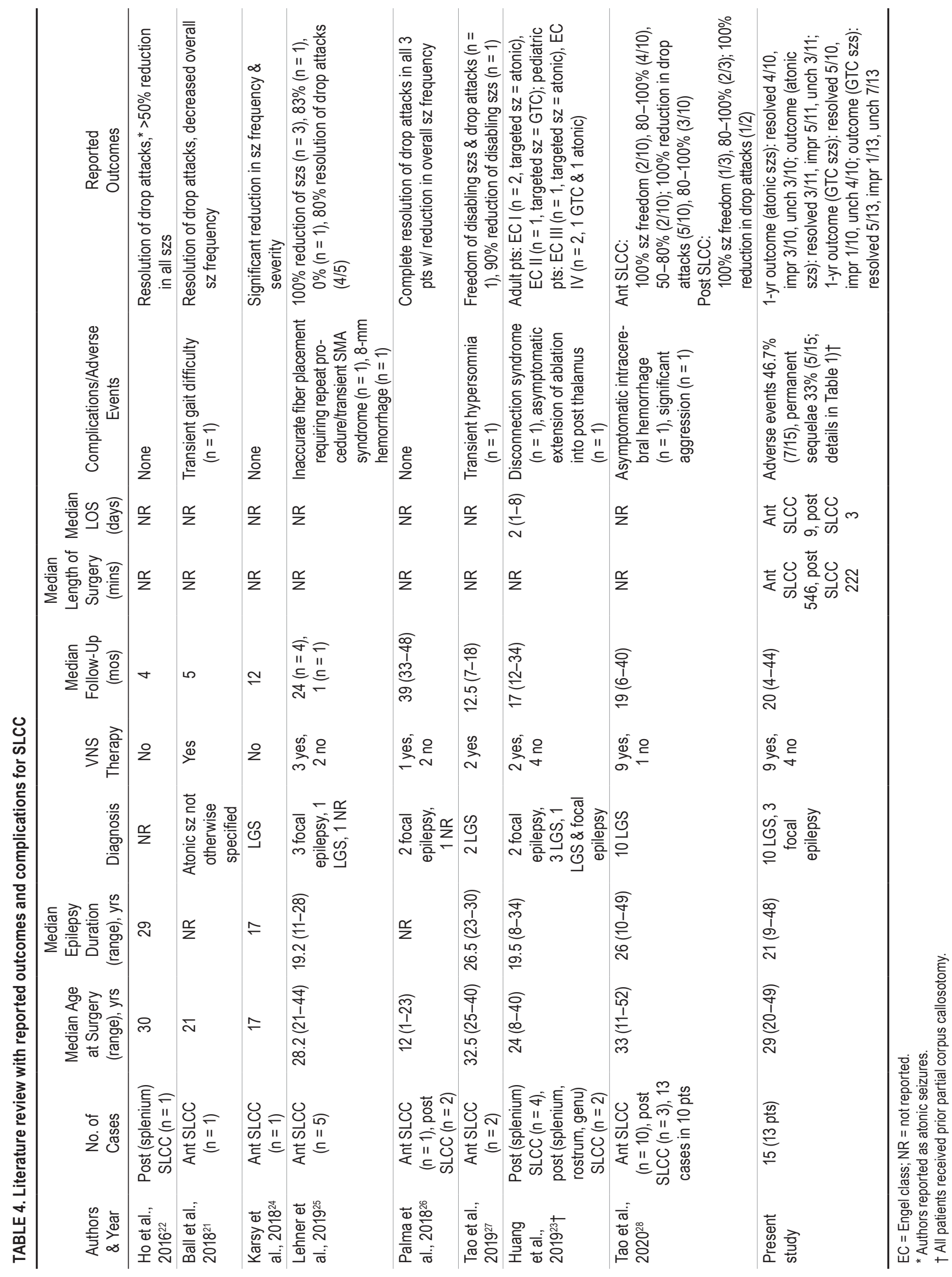




\section{Discussion}

This is the largest reported series of SLCC for epilepsy to date. We report the outcomes of SLCC and comparative accuracies of two distinct stereotactic approaches and their associated workflows. The limitations of this study include a single-center retrospective case-series design, small sample size, and reliance upon patient reporting of seizures.

In 1940, Van Wagenen and Herren ${ }^{2}$ observed a decrease in seizures after damage to the callosum, including patients with partial or complete callosal division. Anterior two-thirds callosotomy may be most effective when transection includes the callosal isthmus, ${ }^{3,7}$ which may relate to the variable but relatively posterior location of human decussating motor fibers compared with nonhuman primates. ${ }^{33-35}$ Neuropsychological studies of patients with callosotomies in the 1970s determined that sectioning of the anterior callosum through the isthmus but sparing the splenium resulted in fewer deficits than with complete callosal sectioning. ${ }^{36-38}$ Thus, anterior callosotomy sparing the splenium is generally the preferred initial approach, with posterior callosotomy being reserved for patients with persistent seizures or for patients with relatively poor baseline functional status.

Extensive experience with open callosotomy indicates that astatic seizures are the most improved: worthwhile $(>50 \%)$ reduction in astatic seizures has been reported in $45 \%$ and $90 \%$ of patients following anterior and total corpus callosotomy, respectively. ${ }^{6-10}$ Likewise, recent case reports and series using MRg-LITT for callosotomy suggest 70\%-100\% reductions in astatic seizures (Table 4). ${ }^{21-}$ ${ }_{28}$ By comparison, we observed incremental seizure benefits from anterior and posterior SLCC procedures in the majority of patients, and $80 \%$ of patients with primarily astatic seizures treated by anterior and/or posterior SLCC experienced meaningful benefit or resolution (Tables 2 and 4). We also observed meaningful reductions in focal and GTC seizures in individual patients, consistent with some open callosotomy reports. ${ }^{39-42}$

SLCC procedures were associated with higher rates of adverse events, particularly transient respiratory failure, than have generally been reported for open callosotomy in children. Notably, we have observed similar rates of transient respiratory failure in our prior limited institutional experience of open anterior corpus callosotomy in adults (R.E.G., unpublished observations). It is unclear whether these findings reflect differences in reporting or whether callosotomy can be more morbid in adults than in children. During SLCC, transgression or indirect collateral injury of dorsal cingulum and/or motor fibers may impact networks associated with arousal, motivation, and/or motor control. Increased attention to protecting such pathways via alternative trajectories might decrease the morbidity of the laser approach. It seems prudent to plan trajectories through the nondominant hemisphere to minimize risks to dominant motor and/or language networks. We also observed one severe hemorrhagic complication during an aborted anterior SLCC. This is the most clinically significant hemorrhage we have observed in a cumulative experience of more than 300 stereotactic MRg-LITT cases (J.T.W. and R.E.G., unpublished observations). The relatively large number of stereotactic trajectories undertaken in anterior
SLCC may contribute to increased morbidity relative to other procedures with fewer trajectories. Despite adverse events, anterior SLCC was associated with an acute hospitalization LOS shorter than what we generally observe with open callosotomy in adults (R.E.G., unpublished observations). While SLCC can be associated with acceptable morbidity, there is room for improvement.

Compared with ROSA, the ClearPoint single-site workflow provided superior stereotactic accuracy. The robotic approach may be associated with an accumulation of stereotactic errors from coregistration procedures (using scalp surface laser reflectance in our cases), deflections of drilling at nonorthogonal angles, and stereotactic bolt inaccuracy ${ }^{43}$ Small superficial deflections can greatly amplify over long trajectories. Indeed, the serious hemorrhage we observed was associated with a $3.5-\mathrm{mm}$ device deflection following the robotic technique. Workflows involving device placement in the operating room followed by subsequent $\mathrm{CT}$ or MR imaging may only allow adequate assessment of accuracy after a device is placed into the brain. If trajectories or ablations prove problematic once the patient has been transferred to the MRI suite, the physician must consider returning to the operating room or aborting aspects of the procedure.

In contrast, use of direct MRI-guided stereotaxy and a single-site workflow provides several advantages (Supplemental Figure). Direct MRI guidance with ClearPoint allows real-time recognition and mitigation of potential inaccuracy at any point before, during, and after placing a device into the brain. Relatedly, ClearPoint mitigates inaccuracies from nonorthogonal drilling, because the laser device is guided and secured by a platform overlying a craniostomy and the trajectory does not directly depend upon the accuracy of the craniostomy or bolt direction. Finally, ClearPoint mitigates interdependence of anticipated trajectories. At any stage in the ablation procedure, subsequent trajectory plans can take into account the results of prior actions. In one case (patient 6), the entire anterior SLCC was performed from a series of trajectories through one craniostomy site because each subsequent trajectory could be planned after directly observing what had been accomplished by prior actions. The predominant disadvantages of the MRI-based procedure are the prolonged use of a diagnostic scanner and limited availability of interventional/intraoperative MRI suites that are located in a limited number of surgical epilepsy centers.

\section{Conclusions}

MRI-guided SLCC is a potentially safe and effective alternative to traditional open corpus callosotomy. In the future, automated trajectory planning algorithms may provide safer, faster, and more consistent trajectory placement. ${ }^{44,45}$ Use of advanced imaging such as tractography could facilitate patient-specific disconnection procedures. Partial callosotomies, particularly of the isthmus, might benefit patients with astatic seizures while further reducing the risks of adverse events and cognitive deficits.

\section{References}

1. Mathews MS, Linskey ME, Binder DK. William P. van Wa- 
genen and the first corpus callosotomies for epilepsy. $J \mathrm{Neu}$ rosurg. 2008;108(3):608-613.

2. Van Wagenen W, Herren RY. Surgical division of commissural pathways in the corpus callosum: relation to spread of an epileptic attack. Arch NeurPsych. 1940;44(4):740-759.

3. Huck FR, Radvany J, Avila JO, et al. Anterior callosotomy in epileptics with multiform seizures and bilateral synchronous spike and wave EEG pattern. Acta Neurochir Suppl (Wien). 1980;30:127-135.

4. Spencer SS, Spencer DD, Sass K, et al. Anterior, total, and two-stage corpus callosum section: differential and incremental seizure responses. Epilepsia. 1993;34(3):561-567.

5. Lancman G, Virk M, Shao H, et al. Vagus nerve stimulation vs. corpus callosotomy in the treatment of Lennox-Gastaut syndrome: a meta-analysis. Seizure. 2013;22(1):3-8.

6. Graham D, Tisdall MM, Gill D. Corpus callosotomy outcomes in pediatric patients: a systematic review. Epilepsia. 2016;57(7):1053-1068.

7. Pinard JM, Delalande O, Chiron C, et al. Callosotomy for epilepsy after West syndrome. Epilepsia. 1999;40(12):1727-1734.

8. Rahimi SY, Park YD, Witcher MR, et al. Corpus callosotomy for treatment of pediatric epilepsy in the modern era. Pediatr Neurosurg. 2007;43(3):202-208.

9. Shimizu H. Our experience with pediatric epilepsy surgery focusing on corpus callosotomy and hemispherotomy. Epilepsia. 2005;46(suppl 1):30-31.

10. Turanli G, Yalnizoğlu D, Genç-Açikgöz D, et al. Outcome and long term follow-up after corpus callosotomy in childhood onset intractable epilepsy. Childs Nerv Syst. 2006; 22(10):1322-1327.

11. Wilson DH, Reeves A, Gazzaniga M. Division of the corpus callosum for uncontrollable epilepsy. Neurology. 1978;28(7): 649-653.

12. Jalilian L, Limbrick DD, Steger-May K, et al. Complete versus anterior two-thirds corpus callosotomy in children: analysis of outcome. J Neurosurg Pediatr. 2010;6(3):257-266.

13. Kasasbeh AS, Smyth MD, Steger-May K, et al. Outcomes after anterior or complete corpus callosotomy in children. Neurosurgery. 2014;74(1):17-28.

14. Stigsdotter-Broman L, Olsson I, Flink R, et al. Long-term follow-up after callosotomy - a prospective, population based, observational study. Epilepsia. 2014;55(2):316-321.

15. Ellis JA, Mejia Munne JC, Wang SH, et al. Staged laser interstitial thermal therapy and topectomy for complete obliteration of complex focal cortical dysplasias. J Clin Neurosci. 2016;31:224-228.

16. McCracken DJ, Willie JT, Fernald BA, et al. Magnetic resonance thermometry-guided stereotactic laser ablation of cavernous malformations in drug-resistant epilepsy: imaging and clinical results. Oper Neurosurg (Hagerstown). 2016;12(1): 39-48.

17. Thompson SA, Kalamangalam GP, Tandon N. Intracranial evaluation and laser ablation for epilepsy with periventricular nodular heterotopia. Seizure. 2016;41:211-216.

18. Willie JT, Laxpati NG, Drane DL, et al. Real-time magnetic resonance-guided stereotactic laser amygdalohippocampotomy for mesial temporal lobe epilepsy. Neurosurgery. 2014; 74(6):569-585.

19. Willie JT, Malcolm JG, Stern MA, et al. Safety and effectiveness of stereotactic laser ablation for epileptogenic cerebral cavernous malformations. Epilepsia. 2019;60(2):220-232.

20. Boerwinkle VL, Foldes ST, Torrisi SJ, et al. Subcentimeter epilepsy surgery targets by resting state functional magnetic resonance imaging can improve outcomes in hypothalamic hamartoma. Epilepsia. 2018;59(12):2284-2295.

21. Ball T, Sharma M, White AC, Neimat JS. Anterior corpus callosotomy using laser interstitial thermal therapy for refractory epilepsy. Stereotact Funct Neurosurg. 2018;96(6): 406-411.
22. Ho AL, Miller KJ, Cartmell S, et al. Stereotactic laser ablation of the splenium for intractable epilepsy. Epilepsy Behav Case Rep. 2016;5:23-26.

23. Huang Y, Yecies D, Bruckert L, et al. Stereotactic laser ablation for completion corpus callosotomy. J Neurosurg Pediatr. 2019;24(4):433-441.

24. Karsy M, Patel DM, Halvorson K, et al. Anterior two-thirds corpus callosotomy via stereotactic laser ablation. Neurosurg Focus. 2018;44(VideoSuppl2):V2.

25. Lehner KR, Yeagle EM, Argyelan M, et al. Validation of corpus callosotomy after laser interstitial thermal therapy: a multimodal approach. J Neurosurg. 2019;131(4):1095-1105.

26. Palma AE, Wicks RT, Popli G, Couture DE. Corpus callosotomy via laser interstitial thermal therapy: a case series. $J$ Neurosurg Pediatr. 2018;23(3):303-307.

27. Tao JX, Issa NP, Wu S, et al. Interstitial stereotactic laser anterior corpus callosotomy: a report of 2 cases with operative technique and effectiveness. Neurosurgery. 2019;85(3): E569-E574.

28. Tao JX, Satzer D, Issa NP, et al. Stereotactic laser anterior corpus callosotomy for Lennox-Gastaut syndrome. Epilepsia. 2020;61(6):1190-1200.

29. Jethwa PR, Barrese JC, Gowda A, et al. Magnetic resonance thermometry-guided laser-induced thermal therapy for intracranial neoplasms: initial experience. Neurosurgery. 2012; 71(1)(Suppl Operative):133-145.

30. Burchiel KJ, McCartney S, Lee A, Raslan AM. Accuracy of deep brain stimulation electrode placement using intraoperative computed tomography without microelectrode recording. J Neurosurg. 2013;119(2):301-306.

31. Malmgren K, Rydenhag B, Hallböök T. Reappraisal of corpus callosotomy. Curr Opin Neurol. 2015;28(2):175-181.

32. Bower RS, Wirrell E, Nwojo M, et al. Seizure outcomes after corpus callosotomy for drop attacks. Neurosurgery. 2013; 73(6):993-1000.

33. Cipolloni PB, Pandya DN. Topography and trajectories of commissural fibers of the superior temporal region in the rhesus monkey. Exp Brain Res. 1985;57(2):381-389.

34. Hofer S, Frahm J. Topography of the human corpus callosum revisited-comprehensive fiber tractography using diffusion tensor magnetic resonance imaging. Neuroimage. 2006;32(3): 989-994.

35. Zarei M, Johansen-Berg H, Smith S, et al. Functional anatomy of interhemispheric cortical connections in the human brain. J Anat. 2006;209(3):311-320.

36. Gazzaniga MS, Risse GL, Springer SP, et al. Psychologic and neurologic consequences of partial and complete cerebral commissurotomy. Neurology. 1975;25(1):10-15.

37. Gordon HW, Bogen JE, Sperry RW. Absence of deconnexion syndrome in two patients with partial section of the neocommissures. Brain. 1971;94(2):327-336.

38. Risse GL, Gates J, Lund G, et al. Interhemispheric transfer in patients with incomplete section of the corpus callosum. Anatomic verification with magnetic resonance imaging. Arch Neurol. 1989;46(4):437-443.

39. Oguni H, Olivier A, Andermann F, Comair J. Anterior callosotomy in the treatment of medically intractable epilepsies: a study of 43 patients with a mean follow-up of 39 months. Ann Neurol. 1991;30(3):357-364.

40. Rathore C, Abraham M, Rao RM, et al. Outcome after corpus callosotomy in children with injurious drop attacks and severe mental retardation. Brain Dev. 2007;29(9):577585.

41. Reutens DC, Bye AM, Hopkins IJ, et al. Corpus callosotomy for intractable epilepsy: seizure outcome and prognostic factors. Epilepsia. 1993;34(5):904-909.

42. Sunaga S, Shimizu H, Sugano H. Long-term follow-up of seizure outcomes after corpus callosotomy. Seizure. 2009; 18(2):124-128. 
43. Brandmeir NJ, Savaliya S, Rohatgi P, Sather M. The comparative accuracy of the ROSA stereotactic robot across a wide range of clinical applications and registration techniques. $J$ Robot Surg. 2018;12(1):157-163.

44. Vakharia VN, Sparks R, Li K, et al. Automated trajectory planning for laser interstitial thermal therapy in mesial temporal lobe epilepsy. Epilepsia. 2018;59(4):814-824.

45. Vakharia VN, Sparks RE, Vos SB, et al. Computer-assisted planning for minimally invasive anterior two-thirds laser corpus callosotomy: a feasibility study with probabilistic tractography validation. Neuroimage Clin. 2020;25:102174.

\section{Disclosures}

Dr. Curry reports being a consultant to Medtronic, Inc. Dr. Gross reports being a consultant to Medtronic, and receiving support of non-study-related clinical or research effort from Medtronic. Dr. Willie reports being a consultant to Medtronic, ClearPoint Neuro, NeuroPace, AIMM Therapeutics, and NICO Medical, and receiving support of non-study-related clinical or research effort from Medtronic, NeuroPace, and NICO Medical.

\section{Author Contributions}

Conception and design: Willie, Rich, Fasano, Curry, Gross.

Acquisition of data: Willie, Rich, Fasano, Isbaine, Saindane, Qiu,
Gross. Analysis and interpretation of data: Willie, Rich, Fasano, Isbaine, Saindane, Qiu, Gross. Drafting the article: Willie, Rich, Fasano. Critically revising the article: Willie, Rich, Fasano, Saindane, Qiu, Curry, Gross. Reviewed submitted version of manuscript: all authors. Approved the final version of the manuscript on behalf of all authors: Willie. Statistical analysis: Rich, Isbaine, Qiu. Administrative/technical/material support: Willie, Rich, Gross. Study supervision: Willie, Rich, Gross.

\section{Supplemental Information}

\section{Online-Only Content}

Supplemental material is available with the online version of the article.

Supplemental Figure. https://thejns.org/doi/suppl/10.3171/ 2020.7.JNS20498.

\section{Correspondence}

Jon T. Willie: Washington University School of Medicine and Barnes-Jewish Hospital, St. Louis, MO. jontwillie@wustl.edu. 